\title{
Clinical performance of a new aspheric dual-optic accommodating intraocular lens
}

\author{
This article was published in the following Dove Press journal: \\ Clinical Ophthalmology \\ 19 November 2014 \\ Number of times this article has been viewed
}

\author{
Eduardo F Marques' \\ António Castanheira-Dinis ${ }^{2}$ \\ 'Department of Ophthalmology, \\ Hospital da Cruz Vermelha, Lisboa, \\ Portugal; ${ }^{2}$ Visual Sciences Research \\ Centre, Universidade de Lisboa, \\ Portugal
}

Purpose: To describe the clinical performance of a new dual-optic intraocular lens (IOL) with an enhanced optic profile designed to mimic natural accommodation.

Patients and methods: Prospective multicenter clinical study with the new dual-optic aspheric accommodating IOL (Synchrony $\mathrm{Vu}$ ) in 74 patients (148 eyes) undergoing cataract surgery. Refractive target was emmetropia. Examinations at 1 month and 6 months included subjective refractions; visual acuities at near, intermediate, and far; mesopic contrast sensitivity with and without glare; safety data; and subjective survey on dysphotopsia (halos and glare).

Results: Clinical data at 6 months showed $89 \%$ of the eyes within $\pm 1.0 \mathrm{D}$ spherical equivalent refraction. Mean binocular uncorrected and distance-corrected visual acuity was 20/20 at far $(0.00 \pm 0.11 \log$ MAR and $-0.06 \pm 0.08 \log$ MAR, respectively $), 20 / 20$ at intermediate $(0.01 \pm 0.13$ $\log \mathrm{MAR}$ and $-0.01 \pm 0.10 \log \mathrm{MAR}$, respectively), and 20/25 at near $(0.10 \pm 0.14 \log \mathrm{MAR}$ and $0.14 \pm 0.15 \log$ MAR, respectively). Mesopic contrast sensitivity was within normal limits. Seventy-eight percent of the patients had no spectacles and $70 \%$ had no dysphotopsia. One eye had IOL repositioning within 1 month of surgery.

Conclusion: The new aspheric Synchrony Vu accommodating IOL provided good visual performance at a range of distances without affecting quality of vision and with minimal safety considerations.

Keywords: accommodating IOL, cataract surgery, intraocular lens, presbyopia

\section{Introduction}

Accommodating intraocular lenses (IOLs) are dynamic devices that need to optimally interact with the capsular bag and ciliary muscle to effect a change in optical power during near viewing. ${ }^{1,2}$ The equilibrium between the IOL and the intraocular accommodative plant (capsular bag, zonular fibers, and ciliary muscle) may ultimately impact performance of the accommodating IOL. In addition, the dynamic nature of accommodating IOLs may also impact refractive outcomes, depending on the baseline state of the IOL in the eye.

The dual-optic Synchrony accommodating IOL (Abbott Medical Optics Inc., Santa Ana, CA, USA) was specifically designed to fill the entire capsular bag and benefit from the accommodative capacity of the lens capsule during near vision. ${ }^{3,4}$ The threedimensional, single-piece, foldable silicone lens has a high plus-powered moving optic attached to a variable minus-powered optic through spring haptics. A preloaded injector facilitates implantation through a small incision. The IOL is designed to provide a single, clear focus over a wide range of viewing distances through active accommodation. When implanted within the capsular bag, the capsular tension exerts a compressive force and decreases the interoptic separation of the IOL to a minimum. This compressed state is the emmetropic state of the IOL. With accommodative action, the zonules relax, releasing the tension on the capsular bag, which results in
Correspondence: Eduardo Ferreira

Marques

Department of Ophthalmology,

Hospital da Cruz Vermelha Portuguesa,

Rua Duarte Galvão 54, 1 549-008 Lisboa,

Portugal

Tel +35I 917507836

Fax +351216091239

Email em.lx@netcabo.pt 
a forward displacement of the anterior optic. Design and clinical performance of the parent dual-optic accommodating IOL (Synchrony) have been discussed in detail in previous publications, including improved near vision, reading ability, and long-term performance..$^{4-7}$

A next generation, aspheric version of the dual-optic IOL (Synchrony Vu; Abbott Medical Optics Inc.) was developed to further mimic the optical changes in the eye during natural accommodation. During natural accommodation, the optical power of the crystalline lens increases along with an increase in negative spherical aberration. ${ }^{8,9}$ The central region of the natural lens exhibits greater change in optical power compared to more peripheral regions. ${ }^{8,9}$ The new generation dual-optic IOL is designed to mimic the spatially variant characteristics of natural accommodation and to further improve the near benefit provided by the dual-optic IOL. It incorporates a blended aspheric design with a central zone of negative spherical aberration smoothly transitioning into a spherical periphery. The purpose of this design is to achieve a selective increase in negative spherical aberration during near viewing associated with pupil constriction, while maintaining good optical quality during mesopic conditions with larger pupil diameter.

The objective of the current multicenter clinical study is to evaluate outcomes following primary implantation of this new aspheric dual-optic accommodating IOL for the visual correction of patients undergoing cataract extraction.

\section{Materials and methods Clinical study design}

A multicenter prospective study that included four sites and five investigators was undertaken. Prior approval by each institutional ethical committee was obtained, and all patients provided informed consent after an explanation of the nature of the study, including potential risks and benefits. The study was conducted in compliance with the Declaration of Helsinki. A total of 148 eyes of 74 patients binocularly implanted with the Synchrony Vu IOL were followed up for a period of 6 months. The study was a feasibility study to evaluate the safety and visual performance of this new dual-optic accommodating IOL in a consecutive series of patients.

\section{The IOL}

Synchrony Vu (model SYNC-200) is a single-piece silicone IOL intended for use following cataract extraction. It comprises a dual-optic system arranged coaxially along the optical axis. The front optic diameter is $5.5 \mathrm{~mm}$ and the back optic diameter is $6 \mathrm{~mm}$. The clear optic zone for both optics is $5 \mathrm{~mm}$ diameter. They are supported by $9.5 \mathrm{~mm}$ long haptic structures extending along opposite directions. Two posterior stabilizers extend laterally from the rear lens and are perpendicular to the direction of the haptic extension, with an overall length of $9.8 \mathrm{~mm}$. In addition, the IOL has a small central aspheric zone $(<2.5 \mathrm{~mm}$ diameter) in the center of the anterior lens. It is available in powers of 16-28 D.

\section{Inclusion and exclusion criteria}

The inclusion criteria were age of at least 40 years at the time of surgery, bilateral age-related cataracts, preoperative corneal astigmatism of 1.5 diopters (D) or less, and requiring Synchrony Vu implantation within the available IOL powers. Preoperative exclusion criteria included history of prior ocular surgery or ocular pathology. Surgical exclusions included traumatic surgery; inability to achieve in-the-bag placement of the dual-optic IOL; irregular, decentered, or large $(>5 \mathrm{~mm}$ diameter) continuous curvilinear capsulorhexis (CCC); and anterior or posterior capsule tear.

\section{Surgical technique}

Surgery was performed by five experienced surgeons in four sites using a standardized protocol. Phacoemulsification was performed in all eyes through a $5 \mathrm{~mm}$ (or smaller) CCC to achieve complete overlap of the anterior optic of the lens. The use of a corneal marker (Fujimoto CCC guide) was required to achieve a small anterior CCC sized under $5 \mathrm{~mm}$ diameter. IOL implantation was achieved with a preloaded injector designed to deliver the lens into the capsular bag through a $3.8 \mathrm{~mm}$ clear corneal incision. Surgeons performed meticulous anterior and posterior capsular bag cleanup to remove epithelial cells in an effort to retard capsular fibrosis. Standard postcataract surgery medications $(0.5 \%$ moxifloxacin, $1 \%$ prednisolone acetate, and $0.5 \%$ ketorolac) were used.

\section{Preoperative evaluation}

All patients underwent standard preoperative assessments, including subjective refraction, intraocular pressure, and anterior and posterior segment evaluations. Preoperative biometry measurements, including keratometry, axial length, and anterior chamber depth measured with the IOLMaster 500 (Carl Zeiss Meditec AG, Jena, Germany), were used to calculate the IOL power using a proprietary formula. The target refraction was always emmetropia.

\section{Postoperative evaluation}

Postoperative examinations were performed at 1-day, 1-week, 1-month, and 6-month time points after surgery. 
The 1-month and 6-month visits included measurements of uncorrected (U) visual acuity (VA) at $4 \mathrm{~m}$ (distance; UDVA), at $80 \mathrm{~cm}$ (intermediate; UIVA), and at $40 \mathrm{~cm}$ (near; UNVA) as well as manifest refraction, distance-corrected VA at distance (CDVA), intermediate (DCIVA), and near (DCNVA). Monocular VAs were obtained at all visits, and binocular VAs were obtained at 6 months. Patients requiring refractive correction were prescribed glasses 6-8 weeks after the second eye surgery. The Early Treatment Diabetic Retinopathy Study (ETDRS) charts under photopic light levels were used for VA measurements.

Subjective questionnaires evaluated spectacle use (options included never, near only, far only, both far and near), frequency of spectacle wear (never, occasionally, most of the time, always) separately for near, intermediate, and far distances, and symptoms of glare, halo, and double vision on a 5-point scale. Additionally, the ease of performing activities (such as reading a magazine, reading a book, reading medicine labels, playing cards, using computer, using cell phone, and driving car) was evaluated on a 7-point scale, which included an option of not applicable if the task was not commonly performed by the patient. The questionnaires were administered at the 6-month time point.

In a subset of 40 patients (80 eyes) in three sites, monocular contrast sensitivity was measured with the OPTEC ${ }^{\circledR}$
6500 with the functional acuity contrast test (FACT) charts (Stereo Optical Co., Inc., Chicago, IL, USA) at 6 months after surgery. Two conditions, with and without a glare source, were tested under mesopic light levels $\left(3 \mathrm{~cd} / \mathrm{m}^{2}\right)$.

\section{Statistical analysis}

Statistical analyses were performed using Minitab (Minitab version 16; PA, USA). Descriptive statistics as means, standard deviation (SD), and confidence intervals were calculated for continuous variables, and percentages were calculated for categorical variables. Normality of the data was confirmed using the Kolmogorov-Smirnov test.

\section{Results Demographics}

The mean \pm SD of patient age was $65.6 \pm 9.2$ years; $38 \%$ were males and $62 \%$ females. Monocular data from 148 eyes were available at 1 month and 6 months; binocular data from 74 patients were available at 6 months.

\section{Refraction and visual acuities}

On average, manifest spherical equivalent refraction was slightly myopic at $-0.60 \mathrm{D}$ at 1 month and improved to within $\pm 0.50 \mathrm{D}$ of the refractive target at the 6-month time point (two-tailed $t$-tests, $P<0.01$; Table 1 ). The percentage

Table I Refraction and monocular and binocular visual acuities

\begin{tabular}{|c|c|c|}
\hline & I month (mean \pm SD) & 6 months (mean \pm SD) \\
\hline Spherical refraction (D) & $-0.28 \pm 0.71$ & $-0.04 \pm 0.68$ \\
\hline Cylindrical refraction (D) & $-0.64 \pm 0.57$ & $-0.60 \pm 0.55$ \\
\hline Spherical equivalent refraction (D) & $-0.60 \pm 0.77$ & $-0.34 \pm 0.70$ \\
\hline \multicolumn{3}{|l|}{ Monocular (logMAR; n=|48) } \\
\hline UDVA & $0.20 \pm 0.19(20 / 32)^{\mathrm{a}}$ & $0.14 \pm 0.18(20 / 28)$ \\
\hline UIVA & $0.13 \pm 0.17(20 / 27)$ & $0.13 \pm 0.17(20 / 27)$ \\
\hline UNVA & $0.22 \pm 0.19(20 / 33)$ & $0.25 \pm 0.19(20 / 36)$ \\
\hline CDVA & $0.00 \pm 0.09(20 / 20)$ & $0.00 \pm 0.09(20 / 20)$ \\
\hline DCIVA & $0.09 \pm 0.14(20 / 25)$ & $0.08 \pm 0.14(20 / 24)$ \\
\hline DCNVA & $0.28 \pm 0.17(20 / 38)$ & $0.29 \pm 0.20(20 / 39)$ \\
\hline \multicolumn{3}{|l|}{ Binocular (logMAR; n=74) } \\
\hline UDVA & & $0.00 \pm 0.11(20 / 20)$ \\
\hline UIVA & & $0.01 \pm 0.13(20 / 20)$ \\
\hline UNVA & & $0.10 \pm 0.14(20 / 25)$ \\
\hline CDVA & & $-0.06 \pm 0.08(20 / / 8)$ \\
\hline DCIVA & & $-0.01 \pm 0.10(20 / 20)$ \\
\hline DCNVA & & $0.14 \pm 0.15(20 / 28)$ \\
\hline
\end{tabular}

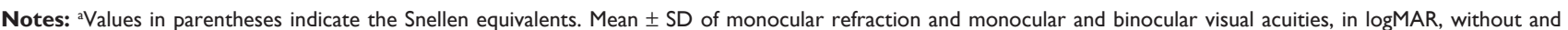
with distance refractive correction at far $(4 \mathrm{~m})$, intermediate $(80 \mathrm{~cm})$, and near $(40 \mathrm{~cm})$ distances. Snellen equivalent of average VA is provided for reference. Refraction was slightly myopic at I month and tended toward emmetropia at 6 months. Monocular distance VA was 20/25 uncorrected and 20/20 with correction. Average monocular VA for intermediate was 20/25 and for near was 20/40 without and with distance refractive correction. Average binocular distance and intermediate VA was 20/20, and binocular near VA was 20/25 without or with refractive correction.

Abbreviations: UDVA, uncorrected distance visual acuity; UIVA, uncorrected intermediate visual acuity; UNVA, uncorrected near visual acuity; CDVA, distance-corrected distance visual acuity; DCIVA, distance-corrected intermediate visual acuity; DCNVA, distance-corrected near visual acuity; SD, standard deviation; VA, visual acuity. 
of eyes with spherical equivalent refraction within $0.50 \mathrm{D}$ of emmetropia was $64 \%$ at the 6-month time point; the refraction within $1.0 \mathrm{D}$ was $89 \%$.

Monocular UDVA improved progressively from the early postoperative period (20/40 at the 1-week time point; data not shown) to a later time (20/25 at 6 months), most likely due to a reduction in some degree of myopia through the early postoperative period.

Monocular CDVA of 20/25 or better was achieved in all eyes. DCIVAs and DCNVAs can be considered a measure of the accommodative or depth of focus performance of the lens. Average monocular DCIVA of 20/25 and DCNVA of 20/40 were achieved by the 1 -month time point and remained stable thereafter up to 6 months.

Binocular UVA (Figure 1A) at 6 months was on average 20/20 for far and intermediate distances and 20/25 at near. DCNVA (Figure 1B) showed a two-line improvement in binocular viewing compared to monocular at 6 months.

\section{Subjective survey questionnaire}

At 6 months, a majority of the patients (78\%) reported not wearing spectacles, followed by $13 \%$ for near only, $6 \%$ for both near and far, and 3\% for far only (Figure 2A). On the question of frequency of spectacle wear for different distances, $94 \%$ reported never wearing glasses for distance, $95 \%$ never for intermediate, and 85\% never for near (Figure 2B).

The ability to perform various activities was evaluated on a 7-point scale from $0-6(0=$ easy and $6=$ difficult $)$, and is shown as the mean $\pm \mathrm{SD}$ of the ratings in Figure 3 . With closer distance or small target size, the mean ratings tended to increase.

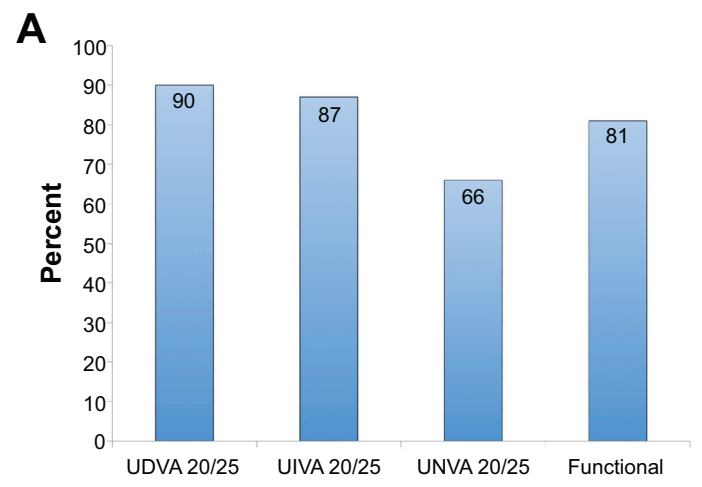

A majority of the patients (69\%) reported experiencing none-to-mild symptoms of glare, halo, or double vision. Some patients experienced glare (21\% moderate and 10\% severe) and halos (18\% moderate and 4\% severe), while 3\% experienced symptoms of double vision (Figure 4).

\section{Contrast sensitivity}

Mesopic contrast sensitivity of Synchrony Vu patients tested both without or with glare (Figure 5), was within the normal limits of the 40-49 years age cohort measured by Hohberger et $a l^{10}$ using the same instrument.

\section{Safety}

None of the study eyes underwent Nd-YAG capsulotomy in the first 6 months after cataract surgery.

IOL optic decentration was noted in three eyes from one site. The UDVA for all the three eyes was 20/20; however, the patients did complain of glare symptoms. All three eyes were monitored throughout the duration of the study without any surgical intervention.

In one eye, the IOL was repositioned within 1 month of surgery due to a partial anterior prolapse of the anterior optic from the capsular bag. The IOL was stable in the bag following repositioning, and at the 6-month visit the patient had a UDVA of 20/32 and CDVA of 20/20.

\section{Discussion}

To evaluate the safety and effectiveness of the new Synchrony $\mathrm{Vu}$ aspheric IOL, a multicenter clinical trial was undertaken. The clinical trial was primarily a feasibility study to evaluate the performance of this new IOL. The

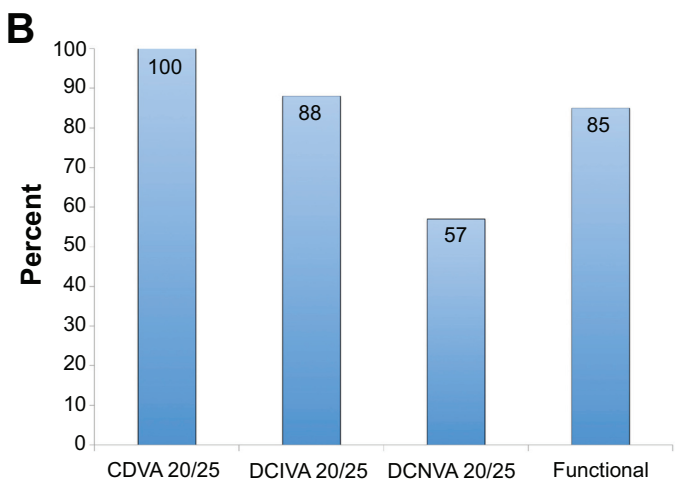

Figure I Uncorrected (A) and distance-corrected (B) binocular visual performance.

Notes: The percentage of patients with binocular uncorrected VA of 20/25 at far (4 m; UDVA), intermediate ( $80 \mathrm{~cm}$; UIVA), and near (40 cm; UNVA) and functional visual acuity metric (ie, combination of 20/25 at far and intermediate and 20/40 at near) are provided for the 6-month time point (A). About $90 \%$ of the patients achieved good far and intermediate VA, and over $65 \%$ of the patients achieved good near VA. Functional visual performance was achieved by more than $80 \%$ of the patients. The percentage of patients with binocular distance-corrected VA of 20/25 at far, intermediate, and near and functional visual acuity metric are also provided for the 6-month time point (B). About $90 \%$ or more patients achieved good far and intermediate VA, and over $55 \%$ of the patients achieved good near VA. Functional visual performance was achieved in over $85 \%$ of the patients.

Abbreviations: VA, visual acuity; UDVA, uncorrected distance visual acuity; UIVA, uncorrected intermediate visual acuity; UNVA, uncorrected near visual acuity; CDVA, distance-corrected distance visual acuity; DCIVA, distance-corrected intermediate visual acuity; DCNVA, distance-corrected near visual acuity. 

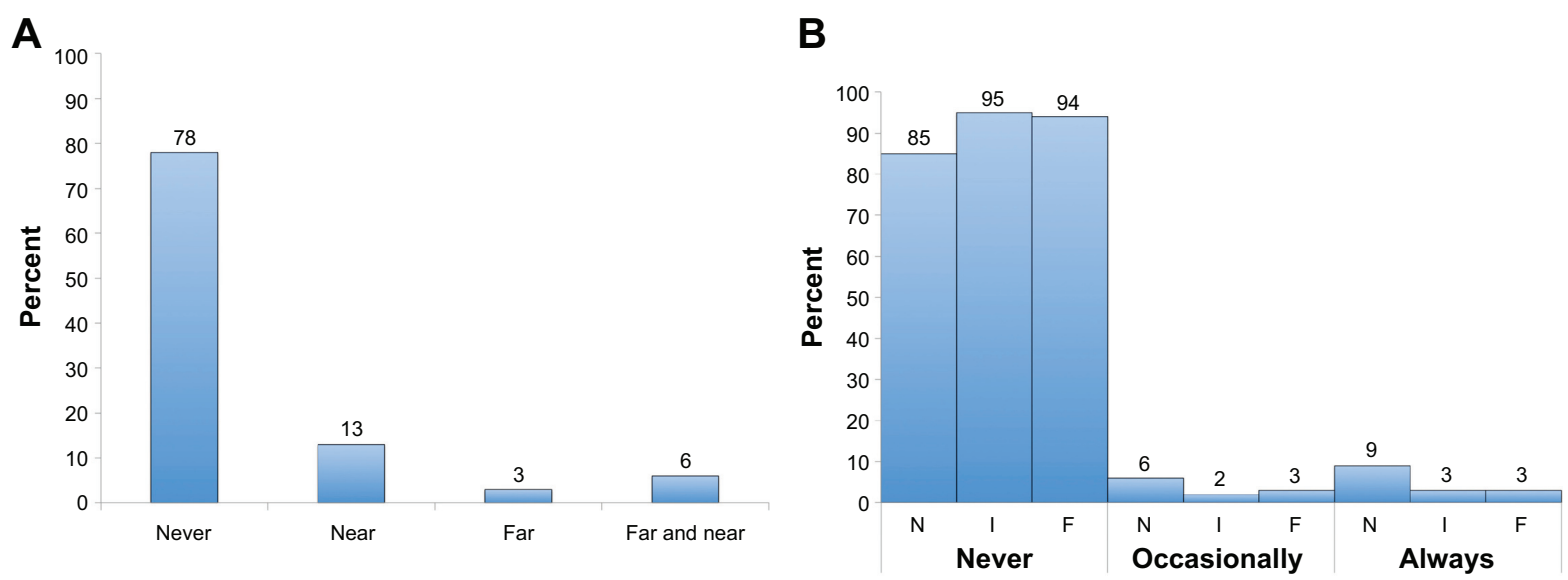

Figure 2 Spectacle independence.

Notes: The responses to the spectacle usage survey on the need for spectacles (rated as never, only near, only far, and both far and near) as percentage are shown (A). The responses to the survey on spectacle need at near $(\mathrm{N})$, intermediate $(\mathrm{I})$, and far $(\mathrm{F})$ distances as percentage are shown (B). Both survey responses are for the 6 -month time point. Almost $80 \%$ of the patients did not require spectacles. Among the patients who needed spectacles, more patients needed glasses for near (I5\%) than for intermediate and far (about 5\%).

lack of a control group can be considered a drawback of the study design, and future studies may be performed with adequate control groups, although the study is strengthened with the use of standardized testing procedures across multiple sites. It was found that following an initial period of myopia, average refractive error close to emmetropia was achieved by the 6-month time point. UDVA largely stabilized by 1 month. It should be noted that all patients were targeted for emmetropia and no intentional monovision was attempted. The initial myopia may be explained by the dual-optics of the Synchrony Vu IOL, designed to allow forward movements of the front optic. The IOL relies on capsular bag forces to maintain the two optics in a compressed state, with a $0.5 \mathrm{~mm}$ separation between the optics for emmetropia. Any forward movement of the front

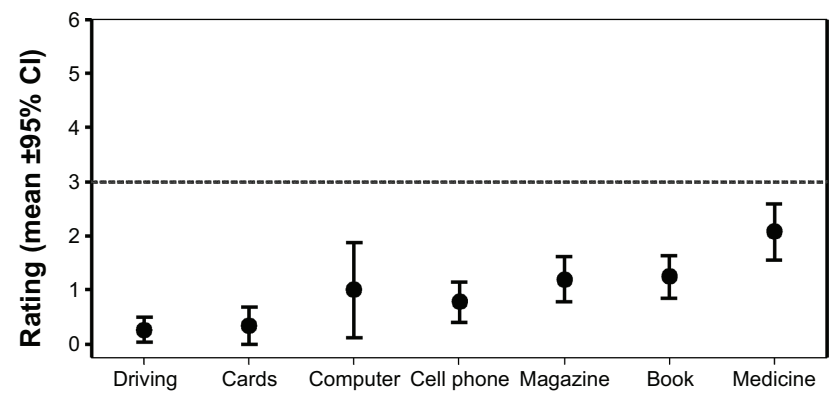

Figure 3 Activity rating.

Notes: The mean $\pm 95 \%$ confidence interval of the survey ratings on various activities scored on a scale from easy (score of 0 ) to difficult (score of 6 ) is shown. Patients also indicated if they did not routinely perform an activity and were excluded from the analysis for that particular activity. The activities were roughly sorted in the order of decreasing target distance or target size (or both). Responses are for the 6-month time point. In general, the average ratings were better than the midpoint (dashed line at " 3 "), with relative ease for far or intermediate tasks and some difficulty for near or fine print tasks.

Abbreviation: $\mathrm{Cl}$, confidence interval. optic will lead to accommodation or, in other words, myopic refractive error. It is likely that in the initial postoperative period, the two optics were separated by greater than $0.5 \mathrm{~mm}$ and gradually compressed as the capsular bag underwent postoperative changes and adapted to the IOL. In general, patients achieved good UDVAs both monocularly (20/25) and binocularly $(20 / 20)$ by 6 months.

IVAs were similar to distance vision (ie, 20/25 monocularly and 20/20 binocularly) with or without distance refractive correction. Similarly, near vision was, on average, $20 / 40$ monocularly and 20/25 binocularly with or without distance refractive correction. It is important to note that distance refractive correction did not reduce intermediate and near visual performance, as might be expected with any monovision strategy.

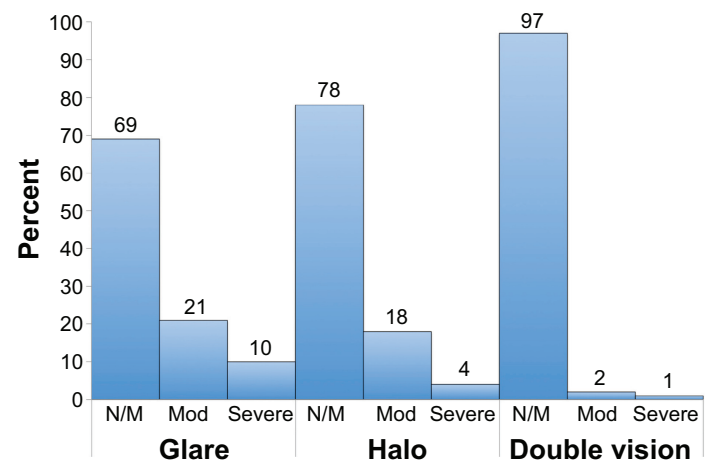

Figure 4 Dysphotopic symptoms.

Notes: Responses to a directed survey on dysphotopsia symptoms of glare, halo and double vision rated on a scale from none (score of 0 ) to very severe (score of 4) are shown as percentages for three categories, namely none or mild symptoms (N/M) (scores of 0 and I), moderate (Mod) (scores of 2), and severe (scores of 3 and 4 ). Responses are for the 6-month time point. About $30 \%$ of the subjects had glare symptoms, $20 \%$ had halo symptoms, and only a few subjects $(<5 \%)$ had double vision symptoms. 
A

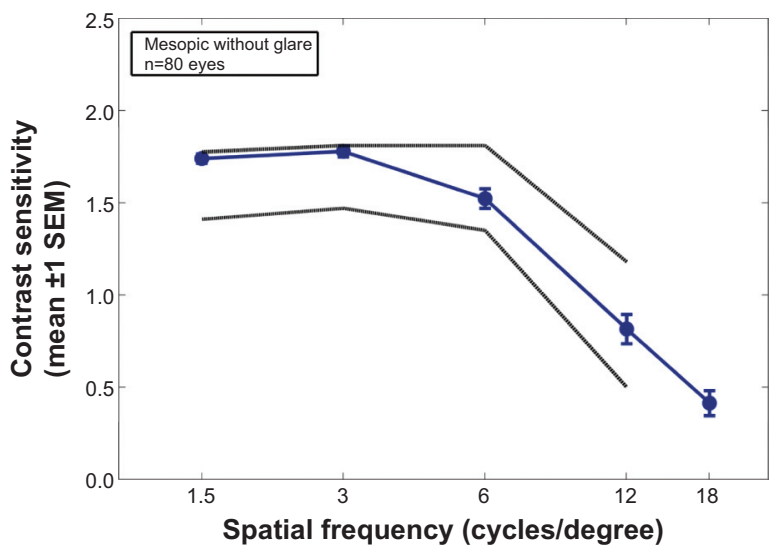

B

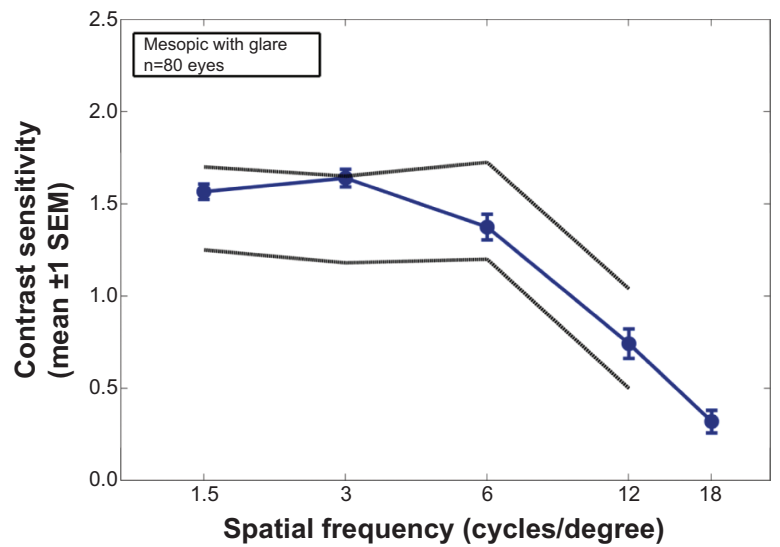

Figure 5 Mesopic contrast sensitivity.

Notes: Monocular mesopic contrast sensitivity data without glare source (A) and with a glare source (B) are shown for the aspheric Synchrony IOL (blue circles). Also shown for reference is the $95 \%$ confidence range of the normative data (gray lines) for the $40-49$ years age group from Hohberger et al ${ }^{10}$ who measured only up to I 2 cycles/ degree using the same Optec 6500 instrument. At all spatial frequencies, Synchrony Vu contrast sensitivities were better than the lower $95 \%$ confidence limit and were also within normal range. Copyright (C) 2007, Springer-Verlag. Springer and Graefes Arch Clin Exp Ophthalmol, 245, 2007, I805-18I4, Measuring contrast sensitivity in normal subjects with OPTEC ${ }^{\circledR}$ 6500: influence of age and glare, Hohberger B, Laemmer R, Adler W, Juenemann AG, Horn FK, Figure 3, with kind permission from Springer Science and Business Media. ${ }^{10}$

Abbreviation: SEM, standard error of the mean.

As a measure of functional performance, it was observed that $80 \%-90 \%$ of the patients achieved a combination of 20/25 VA at distance and intermediate and 20/40 VA at near (Figure 1). If one considers typical newspaper print to be about 20/50, ${ }^{11}$ then these patients can be considered spectaclefree for most day-to-day visual tasks. This is supported by survey data showing $78 \%$ of patients not requiring spectacles (Figure 2). When evaluating ease of performing various tasks, in general the tasks were rated to be easy to moderate, including reading a medicine bottle label (Figure 3 ).

The safety profile of a dual-optic IOL may be expected to be different from a single-optic IOL. It is possible that a dualoptic IOL could experience greater tilt or decentration than a single-optic IOL. The haptic design of an accommodating IOL should be capable of allowing dynamic movements of the IOL as opposed to a single-optic IOL designed for good stability within the capsular bag. Three eyes in the current study were noted to have IOL decentration, with subjective glare symptoms, even though 20/20 VA was achieved in each case. Directed survey on glare, halo, and double vision indicated generally few symptoms. About $30 \%$ of the patients experienced some degree of glare, and $20 \%$ experienced some degree of halo (Figure 4), which is currently better than that reported for multifocal IOLs (up to $70 \%$ ). ${ }^{12,13}$

As further evidence of good imaging quality, mesopic contrast sensitivity with or without glare (Figure 5) was within normal limits. ${ }^{10,14}$

The current clinical study did not include objective accommodation testing because it was designed as a feasibility study with clinically viable test methods. Future studies with Synchrony Vu IOL should consider further optical characterization, objective accommodation testing, and an appropriate control group.

Current options to correct presbyopia during cataract surgery include monovision, multifocal IOLs, and accommodating IOLs. Compared to monovision, multifocal and accommodating IOLs have the potential to maintain binocular function at all distances. The main limitations of multifocal IOLs are the high incidence of dysphotopic phenomena such as glare and halos, ${ }^{15,16}$ and the loss of contrast inherent to a design that causes loss of incident light to higher orders of aberration. ${ }^{17}$ Single-optic accommodating IOLs have in common somewhat limited amplitude of accommodation, ${ }^{18}$ and often provide inadequate or inconsistent near vision. Quality of vision has also been a challenge with single-optic accommodating lenses. ${ }^{19}$ These difficulties led to the development of the dual-optic accommodating IOL in an attempt to provide adequate levels of spectacle independence while maintaining good image quality. The current study showed that overall good visual outcomes can be expected with the Synchrony Vu IOL at intermediate and near distances with or without distance refractive correction.

In conclusion, an aspheric version of the dual-optic accommodating IOL (Synchrony $\mathrm{Vu}$ ) was evaluated in a multicenter clinical trial to show good functional visual performance at a range of distances with minimal visual side effects. The safety profile of an accommodating IOL, especially a dual-optic IOL, is expected to be different from a nonaccommodating single-optic IOL and should be considered together with the benefit. 


\section{Acknowledgments}

The authors thank Ricardo Alarcon, MD, and Victor Bohorquez, MD (Department of Ophthalmology, ServiOftalmos, Bogota, Colombia); Ivan Ossma, MD (Fundación Valle de Lili, Cali, Colombia); Cristina Peris-Martinez, MD, PhD (Fundación Oftalmológica del Mediterráneo, Valencia, Spain) for help in data collection. Sanjeev Kasthurirangan, PhD (Abbott Medical Optics Inc., Santa Ana, CA, USA) for help with data analysis and for writing assistance.

\section{Disclosure}

Eduardo F Marques was consultant and clinical investigator for Abbott Medical Optics during the study. The study was sponsored by Abbott Medical Optics Inc., Santa Ana, CA, USA. The authors report no other conflicts of interest in this work.

\section{References}

1. Charman W. Restoring accommodation: a dream or an approaching reality? Ophthalmic Physiol Opt. 2005;25:1-6.

2. Menapace R, Findl O, Kriechbaum K, Leydolt-Koeppl Ch. Accommodating intraocular lenses: a critical review of present and future concepts. Graefes Arch Clin Exp Ophthalmol. 2007;245:473-489.

3. McLeod SD. Optical principles, biomechanics, and initial clinical performance of a dual-optic accommodating intraocular lens (an American Ophthalmological Society thesis). Trans Am Ophthalmol Soc. 2006;104:437-452.

4. McLeod S, Vargas L, Portney V, Ting A. Synchrony dual-optic accommodating intraocular lens, Part 1: optical and biomechanical principles and design considerations. J Cataract Refract Surg. 2007;33:37-46.

5. Ossma I, Galvis A, Vargas L, Trager MJ, Vagefi MR, McLeod SD. Synchrony dual-optic accommodating intraocular lens, Part 2: pilot clinical evaluation. J Cataract Refract Surg. 2007;33:47-52.
6. McLeod S, Portney V, Ting A. A dual optic accommodating foldable intraocular lens. Br J Ophthalmol. 2003;87:1083-1085.

7. Bohórquez V, Alarcon R. Long-term reading performance in patients with bilateral dual-optic accommodating IOL. J Cataract Refract Surg. 2010;36:1880-1886.

8. Vilupuru A, Roorda A, Glasser A. Spatially variant changes in lens power during ocular accommodation in a rhesus monkey eye. $J$ Vis. 2004;4:299-309.

9. Cheng H, Barnett JK, Vilupuru AS, et al. A population study on changes in wave aberrations with accommodation. J Vis. 2004;4:272-280.

10. Hohberger B, Laemmer R, Adler W, Juenemann AG, Horn FK. Measuring contrast sensitivity in normal subjects with OPTEC ${ }^{\sqrt{R}}$ 6500: influence of age and glare. Graefes Arch Clin Exp Ophthalmol. 2007;245:1805-1814.

11. Oduntan AO. An analysis of print sizes of South African newspapers in relation to prescription of reading devices for low vision patients. S Afr Optom. 2005;64(2):64-70.

12. Summary of Safety and Effectiveness Data Report AcrySof ${ }^{\mathbb{R}}$ ReSTOR $^{\mathbb{R}}$ Apodized Diffractive Optic, 2005. Available at http://www.fda.gov/ ohrms/dockets/dockets/05m0117/05m-0117-aav0001-03-SSED-vol1. pdf. Accessed 18 September 2014.

13. Summary of Safety and Effectiveness Data Report No. Tecnis ${ }^{\circledR}$ Multifocal Posterior Chamber Intraocular Lens, 2009. Available at http:/ www.accessdata.fda.gov/cdrh_docs/pdf8/P080010b.pdf. Accessed 18 September 2014.

14. Pomerance G, Evans D. Test-retest reliability of the CSV-1000 contrast test and its relationship to glaucoma therapy. Invest Ophthalmol Vis Sci. 1994;35:3357-3361

15. Summary of Safety and Effectiveness Data Report No. AcrySof ${ }^{\circledR}$ ReSTOR ${ }^{\circledR}$ Apodized Diffractive Optic, 2005.

16. Summary of Safety and Effectiveness Data Report No. Tecnis Multifocal Posterior Chamber Intraocular Lens, 2009.

17. Pieh S, Marvan P, Lackner B, et al. Quantitative performance of bifocal and multifocal intraocular lenses in a model eye: point spread function in multifocal intraocular lenses. Arch Ophthalmol. 2002;120:23-38.

18. Kuchle M, Nguyen NX, Langenbucher A, et al. Implantation of a new accommodating posterior chamber intraocular lens. J Refract Surg. 2002;18:208-216.

19. Alió JL, Plaza-Puche AB, Montalban R. Near visual outcomes with single-optic and dual-optic accommodating intraocular lenses. J Cataract Refract Surg. 2012;38:1568-1575.
Clinical Ophthalmology

\section{Publish your work in this journal}

Clinical Ophthalmology is an international, peer-reviewed journal covering all subspecialties within ophthalmology. Key topics include: Optometry; Visual science; Pharmacology and drug therapy in eye diseases; Basic Sciences; Primary and Secondary eye care; Patient Safety and Quality of Care Improvements. This journal is indexed on

\section{Dovepress}

PubMed Central and CAS, and is the official journal of The Society of Clinical Ophthalmology (SCO). The manuscript management system is completely online and includes a very quick and fair peer-review system, which is all easy to use. Visit http://www.dovepress.com/ testimonials.php to read real quotes from published authors. 